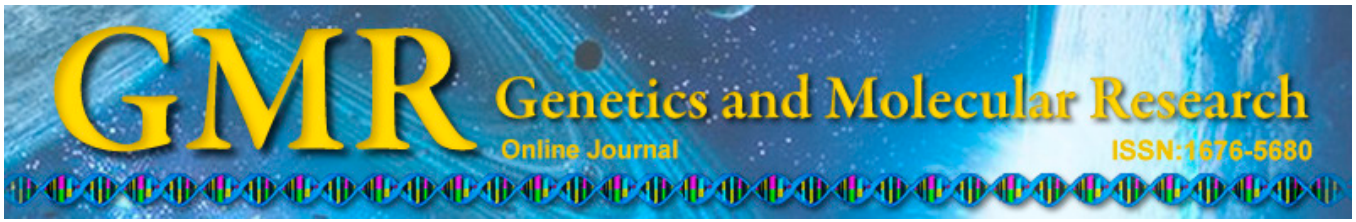

Case Report

\title{
Post-surgical treatment of a patient with ectopic pheochromocytoma
}

\author{
C.F. Sheng, R. Wang, B.Y. Liu, H.M. Zhang, M. Fang and X. Zheng \\ Intensive Care Unit, Taihe Hospital, \\ Affiliated Hospital of Hubei University of Medicine, Shiyan, Hubei, China \\ Corresponding author: $\mathrm{X}$. Zheng \\ E-mail: xiangzhengcn@126.com
}

Genet. Mol. Res. 14 (1): 2139-2145 (2015)

Received July 22, 2014

Accepted November 10, 2014

Published March 20, 2015

DOI http://dx.doi.org/10.4238/2015.March.20.25

\begin{abstract}
We report here the course and treatment of a patient with ectopic pheochromocytoma. The patient was cured after treatment with respiratory and circulatory support, multiple-organ protection, and continuous renal replacement therapy (CRRT) for approximately 2 weeks. After misdiagnosis, a patient with ectopic pheochromocytoma who is being treated should undergo aggressive fluid supplementation and CRRT instead of central venous pressure measurement, which has limited value in guiding fluid supplementation. The main priority is maintaining hypotension. Hypertension may be controlled with rapidacting agents. A good outcome can be anticipated for patients who undergo comprehensive intensive care unit therapy.
\end{abstract}

Key words: Ectopic pheochromocytoma; Misdiagnosis; Retrospective research 


\section{INTRODUCTION}

Ectopic pheochromocytoma is a rare disease (Cohade et al., 2009) that is easily misdiagnosed. Surgery based on a misdiagnosis may cause great difficulties in anesthesia management, and intraoperative and postoperative treatments. Worldwide, few retrospective studies have examined the misdiagnosis of ectopic pheochromocytoma and its post-surgery treatment (Bouasker et al., 2009). Herein, we report on the intraoperative course, treatment, and postoperative intensive care unit (ICU) treatment of a patient with this disease.

\section{CASE REPORT}

A 50-year-old woman was hospitalized on July 22, 2012 with the chief complaint of an abdominal mass for 1 year. Her height and weight were $156 \mathrm{~cm}$ and $67 \mathrm{~kg}$, respectively. She was healthy previously and denied having any diseases such as hypertension.

The physical examination showed the following vital signs: temperature, $36.9^{\circ} \mathrm{C}$; pulse, $82 / \mathrm{min}$; respiration rate, $18 / \mathrm{min}$; and blood pressure (BP), 110/60 $\mathrm{mmHg}$. She was conscious, and no abnormalities were detected on examinations of the heart and lungs. A 15x $15-\mathrm{cm}$ hard mass was palpated in the left lateral distended abdomen; the tumor showed a distinct border from the surrounding tissues.

Laboratory examinations revealed normal findings on blood analysis, as well as normal hepatic/renal function and electrolyte levels. An abdominal ultrasonography scan showed a large heterogeneous peritoneal mass, which was considered to be a giant cystic-solid peritoneal tumor. This was confirmed on a whole-abdomen computed tomography (CT) scan, and a diagnosis of a giant cystic-solid peritoneal tumor with an undetermined nature was established.

The patient underwent an exploratory laparotomy and resection of the tumor under tracheal intubation and inhalation combined with general anesthesia at 11:10 on July 25, 2012.

The patient's BP and heart rate were 140/90 $\mathrm{mmHg}$ and 90 beats per min (bpm), respectively, prior to anesthesia. Exploratory laparotomy did not reveal any abnormalities of the liver, gall bladder, spleen, stomach, small intestine, or colon, except for a giant cystic-solid retroperitoneal tumor $(30 \times 30 \mathrm{~cm})$ with a broad base, which was palpated to be tenacious with fluctuations and limited motion.

During exploration, her BP increased suddenly to $220 / 110 \mathrm{mmHg}$ and heart rate increased to $180 \mathrm{bpm}$ with arrhythmia. The anesthetist and surgeon agreed that the retroperitoneal tumor was likely to be an ectopic pheochromocytoma.

Therefore, the operation was suspended and a rescue was launched. Both central venous and central arterial access were established promptly, and the central venous pressure (CVP) was determined to be $25 \mathrm{cmH}_{2} \mathrm{O}$. Pink frothy sputum aspirated from the trachea and a drop in blood oxygen saturation levels to $78 \%$ suggested acute left heart failure, and the patient was treated accordingly until $3 \mathrm{p} . \mathrm{m}$. At this point, her vital signs were relatively stable with a $\mathrm{BP}$ of $90 / 60 \mathrm{mmHg}$; the operation could then proceed.

The tumor was forced out via an incision after decompression was performed by draining the tumor fluid through a puncture. The envelope at the base of the tumor was separated carefully, and blood vessels of the tumor were ligated; finally, the tumor was removed.

Subsequently, her heart rate dropped abruptly to $60 \mathrm{bpm}$ and BP to $40 / 20 \mathrm{mmHg}$. The operation was suspended again, and epinephrine was injected intravenously while chest compressions were performed, until her vital signs recovered gradually. The operation ended after 
layer-by-layer abdominal closure, and the patient, under an intermittent dose of epinephrine, was admitted to the ICU.

\section{Postoperative Treatment}

At ICU admission, arterial blood gas analysis showed severe metabolic acidosis, routine blood analysis showed slight anemia, electrolyte tests showed hypokalemia and hypocalcemia, and coagulation function tests showed slightly prolonged parameters. Moderate peritoneal effusion was observed on an ultrasonography scan.

The patient's BP remained low after surgery, with a CVP of $32 \mathrm{cmH}_{2} \mathrm{O}$ and a rapid heart rate. Supplementation of blood volume was performed while high doses of epinephrine, dopamine, and dobutamine were administered intravenously to sustain circulation. Anti-fibrinolysis was performed with agents such as p-aminomethyl benzoic acid to prevent disseminated intravascular coagulopathy.

During the night, both her pupils were dilated to $6 \mathrm{~mm}$ and she did not show pupillary light reflex. Given the need for aggressive volume dilatation and liquid supplementation with the presence of pulmonary edema during the operation, aggressive continuous renal replacement therapy (CRRT) was performed along with appropriate ultrafiltration under stabilized circulation to alleviate pulmonary edema and protect the brain from dehydration.

The next day, sustained sedation and assisted aspiration were performed, and the patient's pupil diameter reduced to $2 \mathrm{~mm}$ with a better pupillary light reflex. The patient showed the presence of a large amount of hemosputum. BP was maintained at approximately 140/90 $\mathrm{mmHg}$ by using large doses of vasoactive medications, and her heart rate remained at approximately $90 \mathrm{bpm}$.

On the third day, the patient recovered consciousness, and she was treated intermittently with sedatives and assisted aspiration, despite the presence of a large amount of hemosputum. BP was still maintained at approximately $90 / 60 \mathrm{mmHg}$ with high doses of vasoactive drugs, and her heart rate was approximately $150 \mathrm{bpm}$. Bilateral pulmonary crackles decreased, and cardiac rhythm was regular.

By the fourth day, the volume of the pink frothy sputum decreased significantly compared with that observed previously; therefore, sedative medications were stopped and the parameters of the breathing machine were downregulated. However, high doses of epinephrine along with dopamine administered intravenously were still needed to sustain BP.

Use of the breathing machine was withdrawn successfully on the morning of the fifth day. Tracheal intubation was removed after a chest radiograph showed that the pulmonary edema had ameliorated greatly, and blood gas analysis revealed normal oxygen partial pressure. However, as the patient's BP fluctuated greatly, high doses of norepinephrine and dopamine were required to sustain $\mathrm{BP}$, which had dropped in response to a slightly lowering of the dose.

In the afternoon, the patient had palpitations, chest distress, erubescence of the whole body including the face, a high heart rate, and low BP. Bedside electrocardiography (ECG) showed a ST segment depression of $0.2-0.3 \mathrm{mV}$ in leads V3 to V6. Fortunately, her vital signs recovered and were stable throughout positive volume dilation. Then, ECG was repeated, and it showed that the ST segment depression recovered. Corresponding examinations were carried out, including, cardiac ultrasonography; measurements of levels of brain natriuretic peptide, myocardial enzymes, and troponin; and dynamic monitoring of ECG, all of which ruled out the possibility of a cardiac infarction. 
Instances of abrupt heart rate increases up to $150 \mathrm{bpm}$ were observed repeatedly between days 6 and 12, accompanied by hypotension as low as $60 / 30 \mathrm{mmHg}$, erubescence of the skin over the whole body, and dizziness. BP recovered after the dose of vasoactive medications was increased and appropriate volume dilation was performed. CRRT was stopped on day 6.

By day 13, the patient had stable vital signs, and CT scans showed the postoperative alteration in the abdomen as well as normal findings in both the head and chest. In addition, abdominal ultrasonography revealed no abnormalities. The pathological examination reported ectopic pheochromocytoma.

She recovered completely and was discharged on August 9, 2012, with normal hepatic/renal function, serum glucose levels, electrolyte levels, a BP of 130/70 $\mathrm{mmHg}$, and heart rate of $76 \mathrm{bpm}$.

\section{DISCUSSION}

Pheochromocytoma derives from neuroendocrine cells in the chromaffin tissue outside the adrenal gland (Van Braeckel et al., 2009). The occurrence of pheochromocytoma in the retroperitoneum adjacent to the vertebrae is uncommon, and we report a rare manifestation of a giant retroperitoneal tumor here.

All symptoms of pheochromocytoma are attributable to excess catecholamine in circulation causing the BP to rise, resulting in an increase from mild hypertension to a severe hypertensive crisis, a consistent manifestation of this disease (Robert et al., 2012). Other symptoms include palpitations, anxiety and tremors, and sometimes headache and hidrosis ( $\mathrm{Li}$ et al., 2012).

When reviewing the detailed history of this case, we found that the patient usually experienced palpitations and headache, but these symptoms had not been given due attention.

Therefore, pheochromocytoma should be considered in the differential diagnosis of tumors at organs or tissues with a high occurrence rate of extra-adrenal pheochromocytomas, such as those adjacent to the heart, aorta, urethra, or posterior to the peritoneum, and especially those found in patients with hypertension. The patient's BP in this case met the criteria for a diagnosis of critical hypertension (Madani et al., 2007).

The best approach to diagnose or exclude pheochromocytoma is determination of catecholamine and vanillylmandelic acid levels in 24-h urine samples (Madani et al., 2007). Obviously, it was the insufficient diagnosis and differential diagnosis in this case that led to inadequate preoperative preparation and intraoperative anesthesia. The persistent hypotension during the operation resulted in many more difficulties when trying to maintain the postoperative BP and challenges during ICU treatment. Two recommendations based on our experience in the ICU are as follows: supplementation of circulatory volume should be performed, and CRRT should be adopted if necessary to replace CVP, which has limited value in guiding liquid supplementation.

The prolonged effect of catecholamines in patients with pheochromocytoma may lead to reduction in plasma volume. Recent studies indicate that not only the plasma volume but also that of red blood cells may drop (Li et al., 2012).

Moreover, in 1958, Szakas proposed the concept of catecholamine cardiomyopathy characterized by hypertrophy, edema, focal hemorrhage, intima hypertrophy, and infiltration of inflammatory cells, caused by the direct toxicity of catecholamines on the myocardium. The clinical features of catecholamine cardiomyopathy are similar to those of myocarditis, and 
heart failure and arrhythmia may occur in severe cases. Therefore, these patients may have latent myocarditis (Robert et al., 2012), which might correlate with the shrinkage of myocardial contractility and heart failure that took place in the early stages of the operation, and myocardial ischemia as suggested by the postoperative ST segment variation in this report.

Intraoperative volume dilation is encouraged, even in the presence of hypertension or high CVP, otherwise BP might be hard to maintain intraoperatively, and even harder postoperatively (Latham et al., 2012).

Complications including heart failure and pulmonary edema occurred instantly during the operation in our patient. We considered the coexistence of both interstitial pulmonary edema induced by heart failure and edema of pulmonary alveoli caused by large quantities of enlarged vascular beds, and noted that CVP had limited value in guiding liquid supplementation. Therefore, we recommend that the treatment should be guided by pulmonary edema, evaluated by a minimally invasive test with PiCCO (Chen et al., 2011; Zhang et al., 2013). However, these procedures were not performed as they were not permitted by family members of the patient.

In spite of the therapeutic paradox of aggressive volume dilation to maintain BP versus the correction of pulmonary edema, good results were achieved in this case through active CRRT. This seemed to not only keep the balance in pulmonary effusion, but also prevent multiple-organ injuries caused by toxicity induced by excessive catecholamine levels (Legrand et al., 2013).

$\mathrm{BP}$ should be regulated at the discretion of physicians, while the priority is to relieve hypotension and control hypertension with short-acting agents.

Previous reports suggest that $90 \%$ of pediatric patients with this disease manifest with persistent hypertension, which is also observed in approximately $50 \%$ of adult patients. The main difference between these two groups is caused by over-excretion of either epinephrine or norepinephrine (Latham et al., 2012).

A few patients may show episodic hypotension or shock, which may correlate with tumor necrosis, intra-tumor hemorrhage, sudden cessation of catecholamine release, or a severe cardiac event. This usually results in a poor prognosis (Madani et al., 2007).

In our patient, severe hypotension occurred when the tumor was clamped or excised, which was considered attributable to insufficient blood volume and the short half-life of catecholamine $(<1 \mathrm{~min})$. Usually, it is not the adverse effects of hypertension, but instead the abrupt drop in BP even to 0 , along with a sudden decrease in circulatory catecholamine following removal of the tumor that causes death of patients with pheochromocytoma during surgery.

In this patient, after the tumor was removed, the BP dropped abruptly and was not detectable for approximately $5 \mathrm{~min}$. It did not recover until $1 \mathrm{mg}$ epinephrine was injected instantly through a $\mathrm{T}$ joint and then repeated again after $3 \mathrm{~min}$. Meanwhile, rapid volume dilation was also performed and BP was maintained at 90/60 $\mathrm{mmHg}$.

The author prefers intravenous injections of epinephrine instead of norepinephrine where safety is concerned, because of the risk of agents leaking outside the blood vessels during surgery; also, there is a high potential for necrosis of the hypodermis with norepinephrine, but not with epinephrine (Persad et al., 2012).

Norepinephrine should be used decisively if no response is observed in BP after 2 successive doses of epinephrine. In an ICU setting, instant administration of a high dose of norepinephrine is recommended to maintain BP (De Backer and Scolletta, 2013). The large amount of catecholamine released into circulation, however, causes BP to rise abruptly, with a 
systolic pressure up to $200 \mathrm{mmHg}$ and a similar significant increase in diastolic pressure, accompanied by palpitations, shortness of breath, chest oppression, headache, pale complexion, profuse sweating, and blurred vision; in severe cases, hypertension causes cerebral hemorrhage or pulmonary edema (Latham et al., 2012).

Patients after remission may experience extreme fatigue, asthenia, and erubescence of the facial skin. The episodic onset may be initiated by a sudden change in posture, passion, coughing, defecation, or urination. Considerable individual differences can be observed in the frequency and duration of onset, which do not seem to correlate with tumor size (Madani et al., 2007).

The clinical manifestations observed in our patient over 5 consecutive days reflect the symptoms described above, and were considered attributable to catecholamine excreted by the residual tumor or released because of replacement therapy. Antihypertensive agents should be administered immediately to address an abrupt BP elevation, which should be controlled at 21.3-24.0/13.3-16.0 kPa, but should not be decreased to a very low level, because the hypertension is transient.

Antihypertensive drugs with a short-term effect administered intravenously should be preferred to long-acting ones, which may cause difficulties in maintaining BP because the BP will drop rapidly after removal of the tumor. The best approach to control tachycardia and arrhythmia may be short-acting $\beta$-blockers (esmolol) or lidocaine (Madani et al., 2007; Latham et al., 2012).

In short, inadequate diagnosis prior to surgery and inappropriate doses of adrenergic receptor antagonists resulted in difficulties in anesthesia administration and ICU treatment. However, it is inferred that a good outcome may be anticipated for patients who undergo comprehensive ICU therapy.

\section{ACKNOWLEDGMENTS}

Research supported by the Starting Funds for Graduate Students, Hubei University of Medicine (\#2011QDRZ-20).

\section{REFERENCES}

Bouasker I, Zoghlami A, Samaali I, Mohamed Ali E, et al. (2009). Ectopic pheochromocytoma. Tunis. Med. 87: 98-99.

Chen XK, Li SW, Liu DW, Yang RL, et al. (2011). Effects of central venous pressure on acute kidney injury in septic shock. Zhonghua Yi Xue Za Zhi 91: 1323-1327.

Cohade C, Broussaud S, Louiset E, Bennet A, et al. (2009). Ectopic Cushing's syndrome due to a pheochromocytoma: a new case in the post-partum and review of literature. Gynecol. Endocrinol. 25: 624-627.

De Backer D and Scolletta S (2013). Clinical management of the cardiovascular failure in sepsis. Curr. Vasc. Pharmacol. 11: $222-242$

Latham HE, Rawson ST, Dwyer TT, Patel CC, et al. (2012). Peripherally inserted central catheters are equivalent to centrally inserted catheters in intensive care unit patients for central venous pressure monitoring. J. Clin. Monit. Comput. 26: 85-90.

Legrand M, Darmon M, Joannidis M and Payen D (2013). Management of renal replacement therapy in ICU patients: an international survey. Intensive Care Med. 39: 101-108.

Li XG, Zhang DX, Li X, Cui XG, et al. (2012). Adrenocorticotropic hormone-producing pheochromocytoma: a case report and review of the literature. Chin. Med. J. 125: 1193-1196.

Madani R, Al-Hashmi M, Bliss R and Lennard TW (2007). Ectopic pheochromocytoma: does the rule of tens apply? World J. Surg. 31: 849-854.

Persad EA, Raman L, Thompson MT and Sheeran PW (2012). The use of extracorporeal life support in adolescent amlodipine overdose. Indian J. Crit. Care Med. 16: 204-206.

Robert B, Chivot C, Degremont R, Trouillet N, et al. (2012). Thrombosis of the superior mesenteric vein revealing an 
ectopic pheochromocytoma of the organ of Zuckerkandl. Diagn. Interv. Imaging 93: 625-628.

Van Braeckel P, Carlier S, Steelant PJ, Weyne L, et al. (2009). Perioperative management of phaeochromocytoma. Acta Anaesthesiol. Belg. 60: 55-66.

Zhang Z, Xu X, Yao M, Chen H, et al. (2013). Use of the PiCCO system in critically ill patients with septic shock and acute respiratory distress syndrome: a study protocol for a randomized controlled trial. Trials 14: 32. 